\title{
Identification of candidate methylation-responsive genes in ovarian
}

\section{cancer}

\author{
Laura Menendez ${ }^{1}$, DeEtte Walker ${ }^{2,3}$, Lilya V Matyunina ${ }^{2,3}$, \\ Erin B Dickerson 2,3, Nathan J Bowen ${ }^{2,3}$, Nalini Polavarapu2,3, \\ Benedict B Benigno ${ }^{3}$ and John F McDonald*2,3
}

\begin{abstract}
Address: ${ }^{1}$ Department of Genetics, University of Georgia, Athens, GA 30605, USA, ${ }^{2}$ School of Biology, Georgia Institute of Technology, 315 Ferst Drive, Atlanta, GA 30332, USA and ${ }^{3}$ Ovarian Cancer Institute, 225 North Ave., Atlanta, GA 30332, USA

Email: Laura Menendez - Imenen@uga.edu; DeEtte Walker - lila.walker@biology.gatech.edu;

Lilya V Matyunina - lilya.matyunina@biology.gatech.edu; Erin B Dickerson - erin.dickerson@biology.gatech.edu;

Nathan J Bowen - nathan.bowen@biology.gatech.edu; Nalini Polavarapu - nalini@gatech.edu; Benedict B Benigno - mcgene@gatech.edu;

John F McDonald* - mcgene@gatech.edu

* Corresponding author
\end{abstract}

Published: 25 January 2007

Molecular Cancer 2007, 6:10 doi:10.1186/1476-4598-6-10
Received: 2 August 2006

Accepted: 25 January 2007

This article is available from: http://www.molecular-cancer.com/content/6/1/10

(c) 2007 Menendez et al; licensee BioMed Central Ltd.

This is an Open Access article distributed under the terms of the Creative Commons Attribution License (http://creativecommons.org/licenses/by/2.0), which permits unrestricted use, distribution, and reproduction in any medium, provided the original work is properly cited.

\begin{abstract}
Background: Aberrant methylation of gene promoter regions has been linked to changes in gene expression in cancer development and progression. Genes associated with CPG islands (CGls) are especially prone to methylation, but not all $\mathrm{CGl}$-associated genes display changes in methylation patterns in cancers.

Results: In order to identify genes subject to regulation by methylation, we conducted gene expression profile analyses of an ovarian cancer cell line (OVCAR-3) before and after treatment with the demethylating agent 5 -aza-deoxycytidine (5-aza-dC). An overlapping subset of these genes was found to display significant differences in gene expression between normal ovarian surface epithelial cells and malignant cells isolated from ovarian carcinomas. While $40 \%$ of all human genes are associated with CGls, $>94 \%$ of the overlapping subset of genes is associated with CGls. The predicted change in methylation status of genes randomly selected from the overlapping subset was experimentally verified.

Conclusion: We conclude that correlating genes that are upregulated in response to 5 -aza-dC treatment of cancer cell lines with genes that are down-regulated in cancer cells may be a useful method to identify genes experiencing epigenetic-mediated changes in expression over cancer development.
\end{abstract}

\section{Background}

Gene expression profiling is now a common first approach toward the characterization of molecular changes occurring through cancer development and progression [1]. While recurrent changes in patterns of gene expression are beginning to emerge for a variety of cancers, the causal basis of the observed differences in expression remain to be delineated. One characteristic change associated with many cancers is the down regulation of genes involved in suppressing malignant transformation. 
Down regulation of these "tumor-suppressor genes" may occur, not only by genetic (i.e., nucleotide substitution) changes but also by epigenetic modifications, such as DNA methylation $[2,3]$. DNA methylation largely occurs at cytosines associated with $\mathrm{CpG}$ dinucleotides. $\mathrm{CpG}$ rich regions are known as CpG Islands (CGIs). A CGI has been defined as a region of at least $200 \mathrm{bp}$ with a GC content of $50 \%$ or more, and an observed/expected ratio of CpGs higher than 0.6 [4]. Methylation of CGIs in the promoter region of genes is known to transcriptionally repress those genes [3]. Numerous reports have shown that multiple genes are silenced during cancer progression through hypermethylation of the CGIs. Some examples of genes shown to be silenced in ovarian cancers due to hypermethylation include OPCML, RASSF1A, BRAC1 and $p 16$ [5-7].

Treatment of cancer cell lines with the demethylating agent 5-aza-deoxycytidine (5-aza-dC) leads to changes in gene expression due to the loss of methylation in gene regulatory regions [8-11]. In this study, an ovarian cancer cell line (OVCAR-3) was treated with 5-aza-dC to identify genes regulated in cancer cells by methylation. We compared changes in gene expression patterns in the 5-aza-dC treated cancer cell line with observed differences in patterns of gene expression between normal and cancerous ovarian tissue to identify candidate genes undergoing epigenetic changes during the process of ovarian cancer development. The predicted change in methylation status of genes randomly selected from the list of candidate genes was experimentally verified. Our results indicate that correlating genes that are upregulated in response to 5 -aza-dC treatment of cancer cell lines with genes that are down-regulated in ovarian cancers may be a useful method to identify genes experiencing epigenetic-mediated changes in expression over ovarian cancer development.

\section{Results \\ Gene expression changes in OVCAR-3 in response to 5- aza-treatment}

The ovarian cancer cell line, OVCAR-3, was treated with five $\mu \mathrm{M} 5$-aza-dC to identify genes that display a change in expression in response to changes in methylation. After 72 hours of treatment, RNA was extracted, used to synthesize biotinylated cRNA, and hybridized to Affymetrix Human U133 Plus 2.0 oligonucleotide arrays representing approximately 47,000 transcripts. GC Robust Multiarray Analysis (GCRMA) signal values were obtained from the .CEL files as normalized data in $\log 2$ format. Analysis of variance (ANOVA) was applied to identify differentially expressed genes among the three control and the three 5aza-dC treated samples. A total of 831 genes were differentially expressed $(\mathrm{p}<0.01)$ between the control and the 5 -aza-dC treated cells, of which 465 were upregulated and
366 were down-regulated. Tables 1 and 2 display the 30 genes with the highest fold changes in expression (increase or decrease). A higher fold change in expression was observed for genes upregulated after the treatment possibly due to the direct effect of loss of methylation in gene regulatory regions. Among the genes upregulated after 5-aza-dC treatment were imprinted genes (e.g., H19) and genes previously reported to be transcriptionally repressed due to hypermethylation (e.g., CST6 and SPANX in malignant tissue and MAGEA 1 in non-malignant cells/tissues) [12-14]. Confirmation of the microarray results was carried out by RT-PCR of representative genes that were significantly upregulated in response to the 5-aza-dC treatment [Cystatin 6 (CST6), Caveolin-1 (CAV1), Melanoma antigen family A3 (MAGEA3), Chemokine (C-X-C motif) ligand 6 (CXCL6), Aquaporin3 (AQP3), Stratifin (SFN) and Epithelial membrane protein 3 (EMP3)] (Figure 1).

\section{Methylation pattern of candidate genes}

Genes displaying altered expression levels after 5-aza-dC treatment could have been directly or indirectly affected by loss of methylation. Those genes directly affected by $5-$ aza-dC would be expected to have a methylated CGI in or in proximity to promoter regions. Indirect effects might include the altered expression of loci regulated by genes containing CGIs. To identify genes associated with CGIs, we utilized the CGI identification feature of the USCS Human Genome Browser Gateway. Of the 831 genes differentially expressed after 5 -aza-dC treatment, $74 \%$ are known to be associated with CGIs.

The methylation pattern of the promoter region of five genes associated with CGIs and found to be upregulated after 5-aza-dC treatment (CAV1, CST6, MAGEA3, EMP3 and CXCL6) was examined. With the exception of CXCLG, all have been previously shown to be hypermethylated in other cancers or cancer cell lines [12,15-17].

Figure 2 presents the results of sodium bisulfite sequencing analysis of $\mathrm{CPG}$ rich regulatory regions in each of the five genes. All genes showed extensive methylation of their promoter regions in the untreated ovarian cancer cells (OVCAR-3) and a variable decrease of methylation after 5-aza-dC treatment. CST6,CAV1,EMP3 and MAGEA3 displayed a decrease in methylation ranging from $13 \%$ to $61 \%$ while CXCL6 exhibited no methylation change in the promoter region examined. Variability in the response of different CpG regions to 5-aza-dC has been observed previously $[9,18]$, therefore we can not exclude the possibility that expression of CXCL6 is controlled by the methylation levels of a different region than the one studied here. 
Table I: Upregulated genes after 5-aza-dC treatment of OVCAR cells. Chromosomal location and fold change in gene expression are shown for 5-aza-dC treated/untreated OVCAR 3 cells.

\begin{tabular}{|c|c|c|c|c|}
\hline RefSeq Transcript ID & Symbol & Gene Name & Chr. & Fold Change \\
\hline NM_00135I & DAZL & Deleted in azoospermia-like & $3 p 24$ & 27.52 \\
\hline NM_052957 & ACRC & Acidic repeat containing & $\mathrm{Xq} / 3$ & 27.07 \\
\hline NM_I44594 & FLJ32942 & Hypothetical protein FLJ32942 & $12 q 13$ & 22.73 \\
\hline NM_032858 & MAEL & Maelstrom homolog & Iq24 & 18.75 \\
\hline NM_002364 & MAGEB2 & Melanoma antigen family B, 2 & $\times p 21$ & 18.73 \\
\hline NM_005363 & MAGEA6 & Melanoma antigen family A, 6 & $\mathrm{Xq} 28$ & 16.31 \\
\hline NM_0I 2253 & TKTLI & Transketolase-like I & $X q 28$ & 15.02 \\
\hline NM_023930 & KCTDI4 & Potassium channel tetramerisation domain & ||$q \mid 4$ & 11.34 \\
\hline NM_0I3453 & SPANX & Sperm protein associated with the nucleus, $\mathrm{X}$-linked & Xq27 & 10.99 \\
\hline NM_005362 & MAGEA3 & Melanoma antigen family A, 3 & $\mathrm{Xq} 28$ & 10.56 \\
\hline NM_005335 & HCLSI & Hematopoietic cell-specific Lyn substrate I & $3 q 13$ & 10.41 \\
\hline NM_006228 & PNOC & Prepronociceptin & $8 p 21$ & 9.89 \\
\hline NM_I52578 & FMRINB & Fragile $X$ mental retardation I neighbor & $\times q 27$ & 9.24 \\
\hline NM_005367 & MAGEAI2 & Melanoma antigen family $A, 12$ & $\mathrm{Xq} 28$ & 8.81 \\
\hline NM_003289 & TPM2 & Tropomyosin 2 (beta) & $9 p 13$ & 7.00 \\
\hline NM_080618 & CTCFL & CCCTC-binding factor (zinc finger protein)-like & $20 q 13$ & 6.39 \\
\hline NM_0010II544 & MAGEAII & Melanoma antigen family $\mathrm{A}, \mathrm{II}$ & Xq28 & 6.32 \\
\hline NM_0052I3 & CSTA & Cystatin A & $3 q 21$ & 5.80 \\
\hline NR_002196 & $\mathrm{HI9}$ & $\mathrm{HI} 9$, imprinted maternally expressed untranslated mRNA & IIpI5 & 5.71 \\
\hline NM_032048 & EMILIN2 & Elastin microfibril interfacer 2 & $|8 p| \mid$ & 5.41 \\
\hline NM_I7357| & LOC2553I3 & Hypothetical protein LOC255313 & $\times q 24$ & 5.28 \\
\hline NM_003480 & MFAP5 & Microfibrillar associated protein 5 & $12 p \mid 3$ & 4.79 \\
\hline NM_00I323 & CST6 & Cystatin E/M & $1|q| 3$ & 4.79 \\
\hline NM_00I327 & CTAGIB\&A & Cancer-testis antigen IB/cancer-testis antigen IA & Xq28 & 4.58 \\
\hline NM_0010II548 & MAGEA4 & Melanoma antigen family A, 4 & $\mathrm{Xq} 28$ & 4.48 \\
\hline NM_020826 & SYTI3 & Synaptotagmin XIII & IIpII & 4.37 \\
\hline NM_0I3238 & DNAJCI5 & Dnaj (Hsp40) homolog, subfamily C, member I5 & $13 q 14$ & 4.33 \\
\hline NM_005602 & CLDNII & Claudin II & $3 q 26$ & 4.23 \\
\hline NM_001002915 & IGFL2 & Insulin growth factor-like family member 2 & $19 q 13$ & 4.09 \\
\hline NM_004988 & MAGEAI & Melanoma antigen family A, I (directs expression of antigen MZ2-E) & Xq28 & 3.73 \\
\hline
\end{tabular}

Genes responsive to 5-aza-dC in the OVCAR-3 cell line display decreased expression in ovarian adenocarcinomas We hypothesized that if epigenetic mediated changes in gene expression are occurring in ovarian cancer development, at least a subset of the genes upregulated in response to 5-aza-dC treatment in OVCAR-3 cells should display lower levels of expression in ovarian adenocarcinomas relative to controls. To test this hypothesis, we looked for overlap between genes responsive to 5-aza-dC treatment in the OVCAR-3 cells and genes displaying a significant change in expression between normal ovarian surface epithelium brushings (NOSE) and epithelial adenocarcinoma cells isolated from serous papillary epithelial ovarian tumors (EOC) by laser capture microdissection (LCM). Microarray analysis (Affymetrix U133 Plus 2.0) was performed on eight samples of NOSE brushings and eight LCM collections of epithelial cells from EOC. Differentially expressed genes between these samples were identified as described for the OVCAR-3 cell line experiment. Although a comprehensive analysis of the tissue microarray results will be published elsewhere, the average fold difference in expression levels between NOSE and EOC for the overlapping genes (see below) are presented in Additional File 1.
Of the 831 genes displaying a significant change in expression in response to 5-aza-dC treatment of OVCAR-3 cells, 123 displayed a significant decrease in expression between normal ovarian epithelia and adenocarcinomas [see Additional File 1]. Of these 123 genes, 102 were upregulated and 21 were down-regulated in response to the 5-aza-dC treatment in the OVCAR-3 cell line. Included in the list of 102 genes are genes previously identified as being hypermethylated and down-regulated in cancer (e.g., zinc finger protein 185 in prostate cancer [19], CAV1 in breast cancer [20], and DIRAS3 in ovarian cancer [21]). We thus predicted that the 102 genes upregulated in 5aza-dC treated cells that overlapped with genes down-regulated in EOC were likely to have been so, at least in part, due to hypermethylation. To determine if these 102 candidate genes contained CGIs as our model predicts, we examined sequences in and immediately flanking each of the 102 genes. Ninety-six of the 102 candidate genes (94 $\%$ ) were found to have CGIs. In contrast, it has been estimated that only $40 \%$ of all human genes are associated with CGIs [22].

As an initial experimental test of the model, we randomly selected two genes from the list of candidate genes (CAV 
Table 2: Downregulated genes after 5-aza-dC treatment of OVCAR cells. Chromosomal location and fold change in gene expression are shown for 5-aza-dC treated/untreated OVCAR 3 cells.

\begin{tabular}{|c|c|c|c|c|}
\hline RefSeq Transcript ID & Symbol & Gene Name & Chr. & Fold Change \\
\hline NM_0010138360 & MADILI & MAD I mitotic arrest deficient-like I (yeast) & $7 p 22$ & -4.27 \\
\hline NM_004883 & NRG2 & Neuregulin 2 & $5 q 31$ & -3.95 \\
\hline NM_001001331 & ATP2B2 & ATPase, $\mathrm{Ca}++$ transporting, plasma membrane 2 & $3 p 25$ & -2.88 \\
\hline NM_001719 & BMP7 & Bone morphogenetic protein 7 (osteogenic protein I) & $20 q 13$ & -2.56 \\
\hline NM_032932 & RABIIFIP4 & RABI I family interacting protein 4 (class II) & $|7 q| \mid$ & -2.49 \\
\hline NM_014914 & CENTG2 & Centaurin, gamma 2 & $2 q 37$ & -2.30 \\
\hline NM_013372 & GREMI & Gremlin I, cysteine knot superfamily, homolog & $15 q 13$ & -2.28 \\
\hline XM_373734 & --- & Hypothetical LOC388388 & $3 p 13$ & -2.28 \\
\hline NM_02076I & Raptor & Raptor & $17 q 25.3$ & -2.26 \\
\hline NM_001031693 & HHLA3 & HERV-H LTR-associating 3 & -- & -2.20 \\
\hline NM_003598 & TEAD2 & TEA domain family member 2 & $19 q 13$ & -2.18 \\
\hline NM_022773 & FLJI268I & Hypothetical protein FLJI268I & $16 p \mid 3.3$ & -2.18 \\
\hline NM_004389 & CTNNA2 & Catenin (cadherin-associated protein), alpha 2 & $2 p 12$ & -2.09 \\
\hline XM_032996 & MICAL3 & Microtubule associated monoxygenase, calponin and LIM domain containing 3 & $22 q 11$ & -2.08 \\
\hline NM_000620 & NOSI & Nitric oxide synthase I (neuronal) & $12 q 24$ & -2.06 \\
\hline NM_006567 & FARS2 & Phenylalanine-tRNA synthetase 2 & $6 p 25$ & -1.20 \\
\hline NM_003466 & PAX8 & Paired box 8 & $2 q 13$ & -1.99 \\
\hline NM_006613 & GRAP & GRB2-related adaptor protein-like & $|7 p| \mid$ & -1.97 \\
\hline NM_022138 & SMOC2 & SPARC related modular calcium binding 2 & $6 q 27$ & -1.96 \\
\hline NM_0I7594 & DIRAS2 & DIRAS family, GTP-binding RAS-like 2 & $9 q 22$ & -1.96 \\
\hline NM_021018 & HISTIH3F & Histone I, H3f & $6 p 22$ & -1.92 \\
\hline NM_004192 & ASMTL & Acetylserotonin O-methyltransferase-like & Xp22 & -1.92 \\
\hline NM_0024I2 & MGMT & O-6-methylguanine-DNA methyltransferase & $10 q 26$ & -1.91 \\
\hline NM_001466 & FZD2 & Frizzled homolog 2 (Drosophila) & $|7 q 2|$ & -1.91 \\
\hline XM_49847I & LOCI 47727 & Hypothetical protein LOCI 47727 & $19 p 13$ & -1.90 \\
\hline NM_003562 & SLC25AII & Solute carrier family 25 (mitochondrial carrier; oxoglutarate carrier), member II & $17 p 13$ & -1.88 \\
\hline NM_004565 & PEXI4 & Peroxisomal biogenesis factor 14 & $1 \mathrm{p} 36$ & -1.87 \\
\hline NM_003955 & SOCS3 & Suppressor of cytokine signaling 3 & $17 q 25$ & -1.87 \\
\hline NM_007037 & ADAMTS8 & ADAM metallopeptidase with thrombospondin type I motif, 8 & IIq24 & -1.85 \\
\hline NM_022479 & WBSCRI7 & Williams-Beuren syndrome chromosome region 17 & $7 q 11$ & -1.84 \\
\hline
\end{tabular}

1 and $C X C L 6$ ) and one CGI-associated gene not on the list of candidate genes (EMP3) for sodium bisulfite sequencing analysis. Consistent with the prediction of the model, CAV-1 and CXCL6 were found to be hypermethylated in ovarian cancer relative to control tissues while essentially no difference in EMP3 methylation pattern was detected. The observed fold changes in gene expression between control (NOSE) and ovarian cancer (EOC) samples were significant $(\mathrm{p}<0.01)$ for CAV $1(-30.88)$ and CXCL6 ($4.22)$ [see Additional File 1] but not significant $(\mathrm{p}<0.06)$ for EMP $3(+1.10)$. Consistent with these relative changes in levels of gene expression, the relative increase in levels of methylation was greater for Cav 1 than CXCLG and minimal for EMP3 (Figure 3).

\section{Discussion}

The potential contribution of epigenetic changes to cancer development and progression is a topic of much recent interest [e.g., [23-25]]. For example, several studies have implicated changes in DNA methylation with changes in the expression of a number of putative tumor suppressor and oncogenes in a variety of cancers [e.g., $[2,12-21]]$. In an effort to establish a more global perspective of genes that may be prone to changes in DNA methylation during cancer development, a number of investigators have carried out gene profile analyses of a variety of cancer cells treated with demethylating agents (e.g., 5-aza-dC) [e.g., [8-11,26-28]]. The rationale is that genes that are transcriptionally repressed in cancer cells due to hypermethlyation may be released from such constraint after treatment with demethylating agents and thereby identified by a significant increase in gene expression. If valid, such a strategy could be useful in identifying a candidate subset of methylation-regulated genes among the thousands of genes that are typically found to be down-regulated during cancer development. While intuitively appealing, such a strategy rests upon the questionable assumption that all or most genes displaying a significant increase in expression after treatment with demethylating agents are directly responding to an induced change in methylation. The purpose of our study was to conduct a preliminary evaluation of the validity of this assumption and of the overall utility of this approach within the context of ovarian cancer.

We selected for our study the ovarian cancer cell line OVCAR 3 because we had previously determined that it displays a gene expression profile most similar to that of ovarian cancer epithelial cells (data not shown). Comparing the gene expression profile of OVCAR 3 cells before and after treatment with 5-aza-dC revealed a significant ( $\mathrm{p}$ $<0.01)$ change in expression for 831 genes. We deter- 


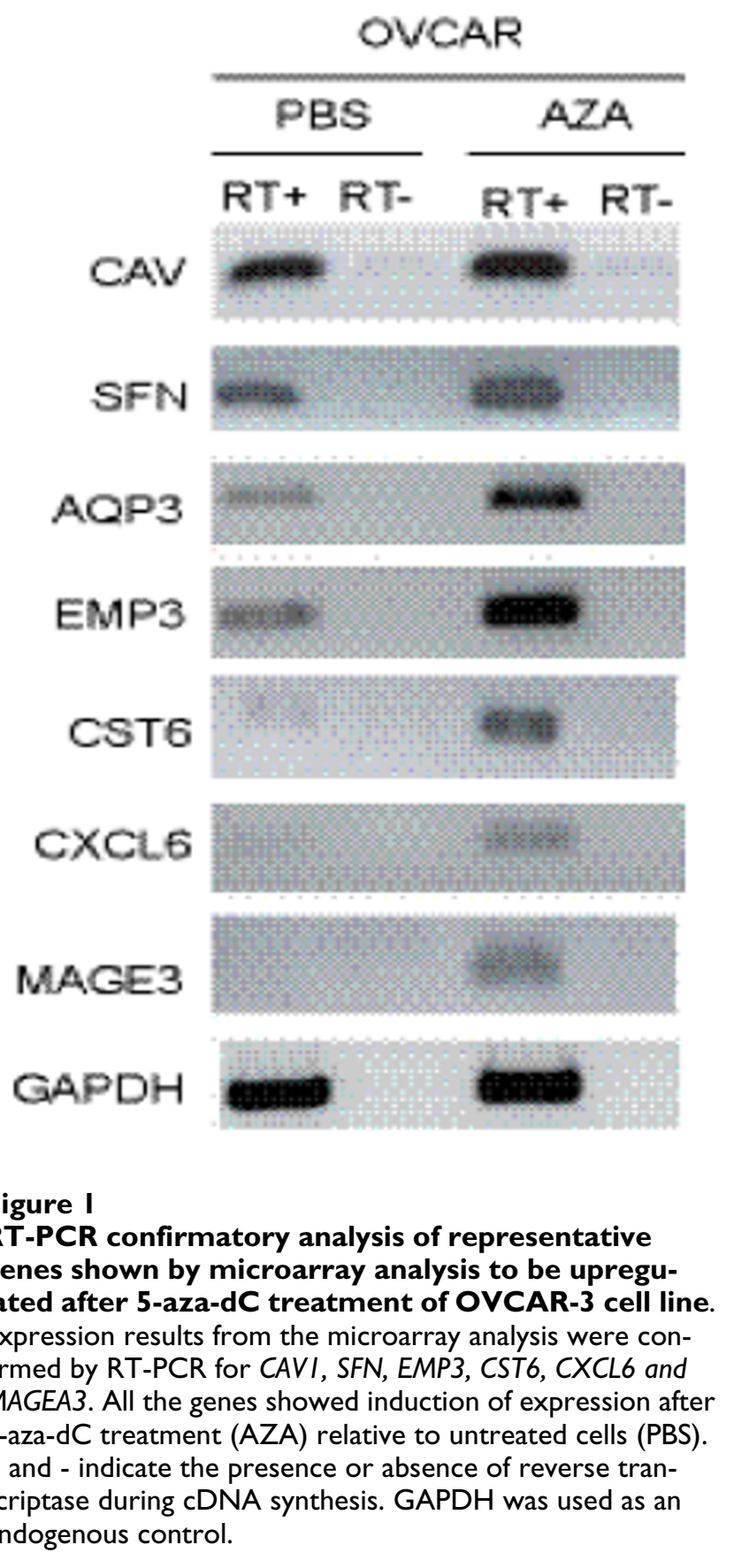

mined that 615 of these 831 genes (74\%) are associated with CpG islands (CGIs). This is a substantially higher fraction than what has been estimated for the human genome overall $(40 \%)$ [22], and suggests that, as expected, CGI-associated genes are especially sensitive to 5 -aza-dC treatment. Only slightly more than half (465 or $56 \%$ ) of the 8315 -aza-dC-responsive genes were significantly upregulated after 5 -aza-dC treatment as would be expected if they had been hypermethylated in the cancer
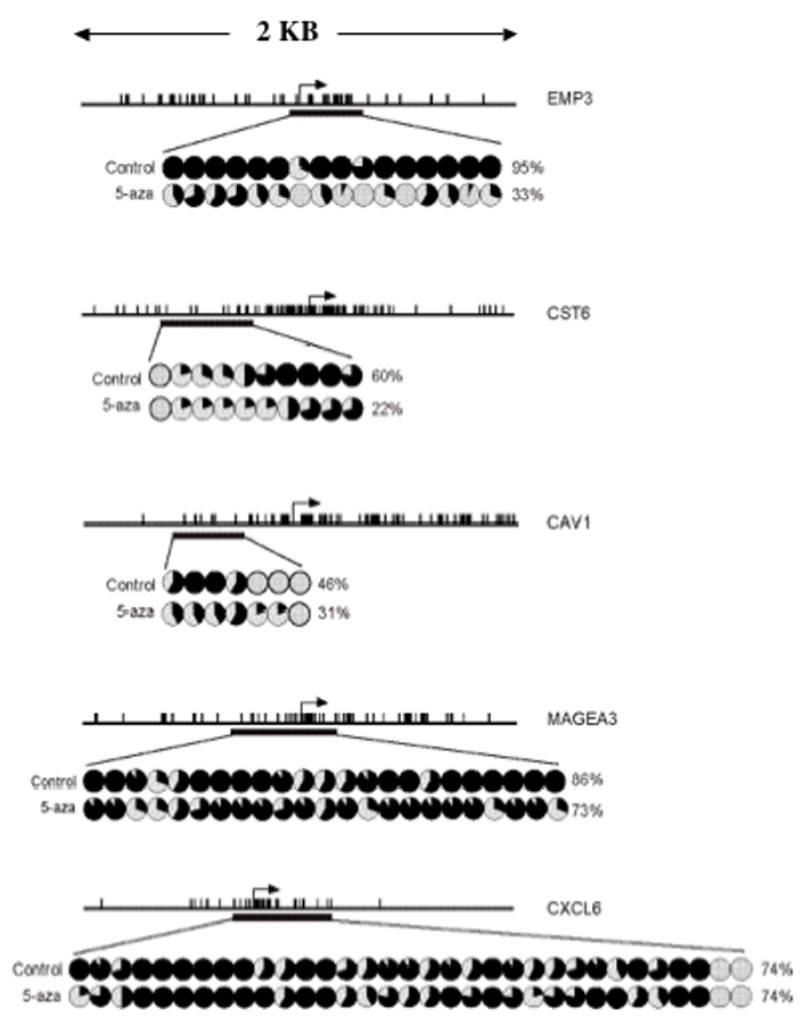

Figure 2

Methylation analysis of genes upregulated after 5aza-dC treatment of the OVCAR-3 cell line. Summary of sodium bisulfite sequencing analysis of $C G$ s associated with the EMP3, CST6, CAVI, MAGEA3 and CXCL6 genes in 5aza- $\mathrm{dC}$ treated and untreated (control) OVCAR-3 cells. Five clones were sequenced for each sample, and each circle displays the percent methylation of all clones for a single CpG dinucleotide (open circle, 100\% unmethylated; filled circle, $100 \%$ methylated). Average percent methylation across all CpG sites for untreated (control) and 5-aza-dC treated cells is shown next to each row of circles. (Vertical bars, CpG dinucleotides; arrow, position of the transcription start site; horizontal black rectangle, region amplified and sequenced after sodium bisulfite).

cell. The explanation as to why the remaining 366 genes displayed a significant decrease in expression after 5-aza$\mathrm{dC}$ treatment is unknown but could, in part, be due to hypomethylated $\mathrm{CpG}$-associated genes being accessed by repressor proteins or to indirect regulatory effects (e.g., non-CpG-associated genes responding to an upregulated repressor protein) or to some unknown gene toxic effect of the treatment. Regardless, we can conclude that up to nearly half of the 5 -aza- $\mathrm{dC}$ responsive genes in the OVCAR 3 cells may not have been directly responding to 5-aza-dC-induced hypomethylation. On the other hand, support for the hypothesis that the majority of those genes upregulated in the cell line after treatment are directly 

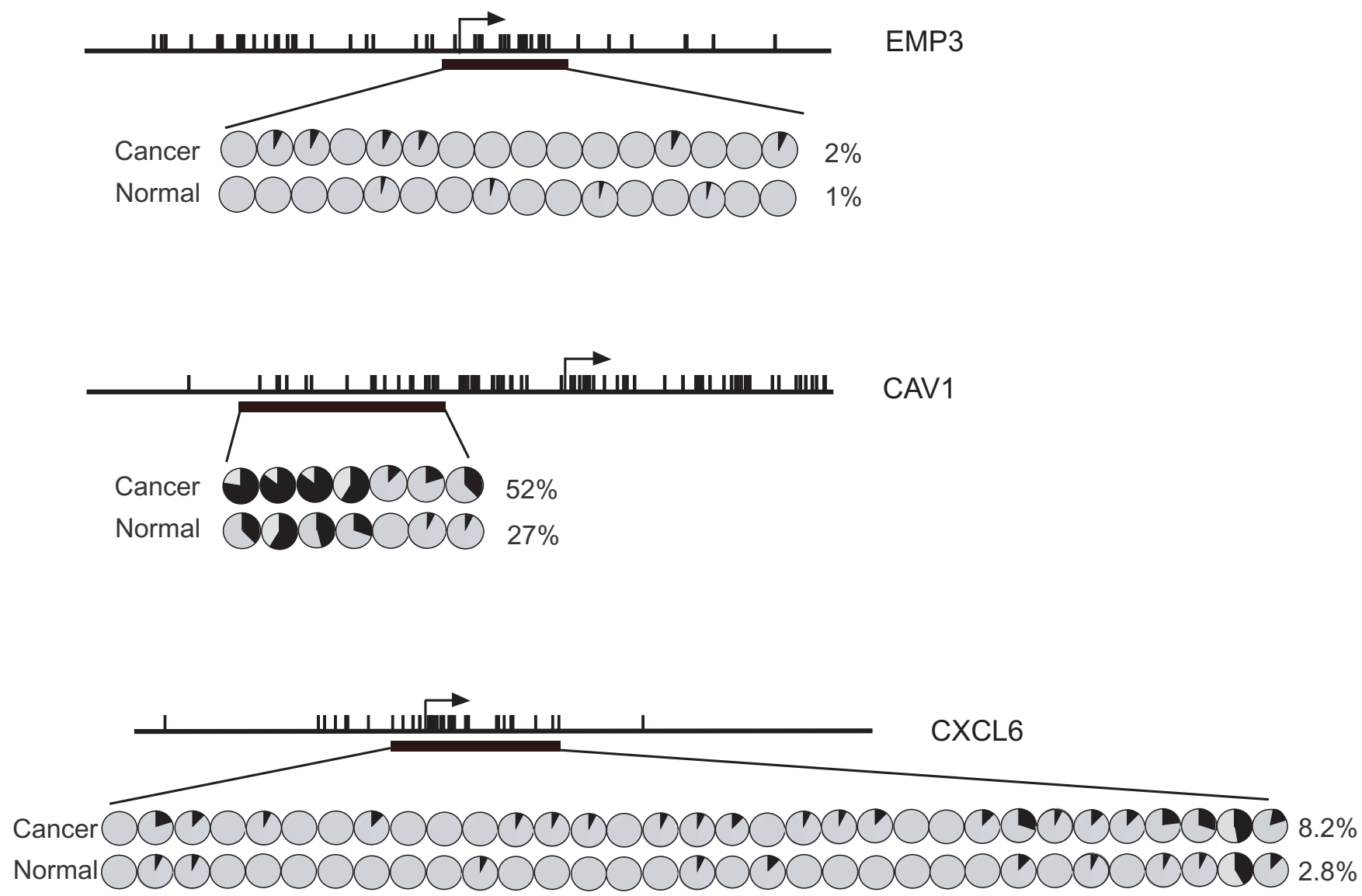

\section{Figure 3}

Methylation analysis of the EMP3, CAVI and CXCL6 genes in ovarian cancer (EOC) and control (NOSE) tissues. Summary of sodium bisulfite sequencing analysis of CGIs associated with the EMP3, CAVI and CXCL6 genes in ovarian ovarian cancer and control (NOSE) tissues. For EMP3, 16 clones for 4 cancer samples and 23 clones for 5 control (NOSE) samples were sequenced. For CAV I, I 7 clones for 4 cancer samples and 18 clones for 4 control (NOSE) samples were sequenced. For CXCL6, 19 clones for 4 cancer samples and 24 clones for 5 normal (NOSE) samples were sequenced. Each circle displays the percent methylation of all clones for a single CpG dinucleotide (open circle, $100 \%$ unmethylated; filled circle, $100 \%$ methylated). Average percent methylation for cancer (EOS) and control (NOSE) samples is shown next to each row of circles. (Vertical bars, CpG dinucleotides; arrow, position of the transcription start site; horizontal black rectangle, region amplified and sequenced after sodium bisulfite).

responding to 5-aza-dC-induced hypomethylation comes from the fact that 5 of the 6 upregulated genes that we directly tested were indeed hypomethylated in response to the 5-aza-dC treatment.

To evaluate the possible relevance of the results from the cell line experiment to actual changes in gene expression that occur during ovarian cancer development, we correlated the OVCAR 3 expression profiles with those from an ovarian cancer tissue analysis recently completed in our laboratory. We reasoned that if methylation dependent changes in gene expression are occurring in ovarian cancer development, at least a subset of the genes upregulated in response to 5-aza-dC treatment in OVCAR 3 cells should display lower levels of expression in ovarian cancer cells relative to controls. Of the 465 genes upregulated in the OVCAR 3 experiment, 102 (22\%) were found to be downregulated in the ovarian cancer cells isolated from tumor samples. Since 102 genes is a small fraction ( $0.02 \%)$ of the $\sim 5000$ genes that were down-regulated in ovarian cancer cells and that we estimate that nearly half of these are associated with CGIs (data not shown), it seems unlikely that monitoring the gene expression response of OVCAR 3 cells to 5 -aza-dC is an effective way to identify all candidate genes that may experience functionally significant changes in methylation over cancer development. How- 
ever, we believe the approach may be useful in identifying a subset of genes that are most likely to experience functionally significant changes in methylation over cancer development. This approach can provide researchers with a useful method by which to identify a candidate set of genes for detailed epigenetic analysis. Our methylation analysis of randomly selected genes from the list of 102 overlapping genes, supports our conclusion.

\section{Conclusion}

We conclude that correlating genes that are upregulated in response to 5-aza-dC treatment of cancer cell lines with genes that are down-regulated in cancer cells may be a useful method to identify genes experiencing epigeneticmediated changes in expression over cancer development.

\section{Methods}

\section{Cell lines and cell culture}

OVCAR-3 cells were grown in RPMI 1640 supplemented with $20 \% \mathrm{v} / \mathrm{v}$ heat-inactivated FBS (Invitrogen, Carlsbad, CA), 2 mM L-glutamine (Mediatech Inc., Herndon, VA), $10 \mathrm{mM}$ HEPES buffer (Sigma, St. Louis, MO) penicillin (100 U/ml), streptomycin $(100 \mu \mathrm{g} / \mathrm{ml}), 1 \mathrm{mM}$ sodium pyruvate (Mediatech Inc.), and $0.01 \mathrm{mg} / \mathrm{ml}$ bovine insulin (Sigma).

\section{5-aza-dC treatment}

OVCAR- 3 cells were plated at a concentration of $1 \times 10^{6}$ in $10 \mathrm{~cm}^{3}$ cell culture dishes. Cells were allowed to adhere to the plates overnight, and the medium was replaced the next morning. Five $\mu \mathrm{M}$ 5-aza-deoxycytidine (Sigma) or PBS was added to the medium and the plates were incubated for 3 days at $37^{\circ} \mathrm{C}$ in a $5 \% \mathrm{CO}^{2}$ atmosphere. Each treatment was done in triplicate.

\section{Microarray}

OVCAR-3 cells were washed once in the plate with HBSS prior to the extraction of RNA and DNA by established techniques (Trizol). The integrity of the RNA was verified using an Agilent 2100 Bioanalyzer (Agilent Technologies, Palo Alto, CA). Biotin labeled cRNA was synthesized, hybridized to Affymetrix U133 Plus 2.0 oligonucleotide arrays and analyzed with a GeneChip Scanner 3000 (Affymetrix, Santa Clara, CA).

\section{Microarray data analysis}

CEL files generated by the Affymetrix Gene Chip Operating System (GCOS) were converted to expression level values using the GCRMA version1.1.0 (ref. below) package implemented from within the Spotfire Decisionsite for Microarray Analysis (DSMA). Probe set intensities

Table 3: Primers used for RT-PCR and Sodium Bisulfite sequencing

\begin{tabular}{|c|c|c|c|c|c|}
\hline Gene & Primer & Sequence & Size & $\mathrm{Tm}$ & Ref. \\
\hline \multirow[t]{4}{*}{ CAVI } & RT-PCR_F & 5'-CGACCCTAAACACCTCAACGATG-3' & 278 & $55^{\circ} \mathrm{C}$ & [15] \\
\hline & RT-PCR-R & 5'-GCAGACAGCAAGCGGTAAAACC-3' & & & \\
\hline & MOD_F & 5'-TGTGTATTTTGTAAATATGGTATAATTTG-3' & 537 & $58^{\circ} \mathrm{C}$ & [15] \\
\hline & MOD_R & 5'-САTTTTCCCTACTCTAAACCAC-3' & & & \\
\hline \multirow[t]{2}{*}{ SFN } & RT-PCR_F & 5'-GTGTGTCCCCAGAGCCATGG-3' & 161 & $60^{\circ} \mathrm{C}$ & [23] \\
\hline & RT-PCR-R & 5'-ACCTTCTCCCGGTACTCACG-3' & & & \\
\hline \multirow[t]{4}{*}{ CST6 } & RT-PCR_F & 5'-AAGACCAGGGTCACTGGAGA-3' & 163 & $60^{\circ} \mathrm{C}$ & [12] \\
\hline & RT-PCR-R & 5'-CGGGGACTTATCACATCTGC-3' & & & \\
\hline & MOD_F & 5'-GGTTGGAATGTTGTAGTGGT-3' & 430 & $55^{\circ} \mathrm{C}$ & [12] \\
\hline & MOD_R & 5'-CCCCAACAACAAATACCAA-3' & & & \\
\hline \multirow[t]{4}{*}{ MAGEA3 } & RT-PCR_F & 5'-TGGAGGACCAGAGGCCCCC-3' & 725 & $68^{\circ} \mathrm{C}$ & [16] \\
\hline & RT-PCR-R & 5'-GGACGATTATCAGGAGGCCTGC-3' & & & \\
\hline & MOD_F & 5'-AGATTTGGTTTGAGGGGAGTAGAAGT-3' & 405 & $60^{\circ} \mathrm{C}$ & [16] \\
\hline & MOD_R & 5'-AACCC(G/A)ACAACAAAAACAACACTAAA-3' & & & \\
\hline \multirow[t]{4}{*}{ EMP3 } & RT-PCR-F & 5'-TGCTCTCССТСАТTCTCTGCTGTC-3' & 274 & $60^{\circ} \mathrm{C}$ & \\
\hline & RT-PCR-R & 5'-CGCTTCCGTAGGTGGATGTAGATG-3' & & & \\
\hline & MOD-F & 5'-TAGTATATATTGAGAGGAGGAGAG-3' & 340 & $62^{\circ} \mathrm{C}$ & [17] \\
\hline & MOD-R & 5'-СТTCCCAAACTACTACATTCCCA-3' & & & \\
\hline \multirow[t]{4}{*}{ CXCL6 } & RT-PCR-F & 5'-CCTGAAGAACGGGAAGC- 3' & 135 & $60^{\circ} \mathrm{C}$ & \\
\hline & RT-PCR-R & 5'-GACTGGGCAATTTTATGATG-3' & & & \\
\hline & MOD-F & 5'-GAGGGATGAATGTAGATAAAGGGAGT-3' & 616 & $60^{\circ} \mathrm{C}$ & \\
\hline & MOD-R & 5'-AACTTCCAAATCCAAACAAACTTACTT-3' & & & \\
\hline \multirow[t]{2}{*}{ GAPDH } & RT-PCR-F & 5'-GAAATCCCATCACCATCTTCCAG-3' & 312 & $56^{\circ} \mathrm{C}$ & \\
\hline & RT-PCR-R & 5'-ATGAGTCCTTCCACGATACAAAAG-3' & & & \\
\hline
\end{tabular}

Size, product size in bp; Tm, annealing temperature 
were filtered with DSMA using a modulation threshold of 5 to include only those probe sets with at least a log base 2 expression value of $\geq 5$. Differentially expressed probe sets were identified using the t-test function of the Profile Anova Tool of DSMA. SAM (Significance Analysis of Microarrays v.2.21) was used to calculate fold change values for the differentially expressed probe sets [29]. Annotations for probe sets were obtained from the NetAffx website [30].

\section{RT-PCR analysis}

$1 \mu \mathrm{g}$ of total RNA was reverse transcribed using oligo (dT) primers from the Superscript III cDNA synthesis kit (Invitrogen). PCR was performed in an Opticon 2 (MJ Research) using $1 \mu$ l of synthesized cDNA. Primer sequences and expected length of the products are provided in Table 3. Gene expression was normalized to the expression of the housekeeping gene GAPDH.

\section{DNA methylation analysis}

Genomic DNA, from 5-aza-dC treated and control OVCAR 3 cells and NOSE brushings and ovarian cancer tissues, was treated with sodium bisulfite to convert unmethylated cytosines to thymines. The EZ DNA Methylation-Gold Kit (Zymo, Orange, CA) was used to modify $500 \mathrm{ng}$ to $1 \mu \mathrm{g}$ of DNA. PCR conditions and primer sequences are provided in Table 3. PCR fragments were gel extracted using the Tissue DNA Extraction Kit (Qiagen) and cloned into pCR 2.1-TOPO TA cloning vectors (Invitrogen). For the OVCAR 3 treated and untreated cell lines, 5 clones each were extracted using the Qiaprep Spin Miniprep Kit (Qiagen) and sequenced (University of Georgia, DNA sequencing Facility). Eighteen to 24 clones from $4 \times$ 5 NOSE (control) samples and 16-19 clones from four cancer samples were sequenced by High-Throughput Sequencing Solutions at the University of Washington.

\section{Competing interests}

The author(s) declare that they have no competing interests.

\section{Authors' contributions}

LM carried out the methylation analyses and RNA extractions of OVCAR-3 cells, participated in the 5-aza-dC treatments and the microarray data analyses and drafted the manuscript. DW carried out the methylation analyses on normal (NOSE) and ovarian cancer (EOC) samples and assisted in the draft of the manuscript. LVM conducted the microarray analyses, EBD participated in the cell culture and 5-aza-dC treatments. NJB contributed to the design of the study and participated in the microarray data analyses. NP contributed to the bioinformatics analysis of the data. BBB provided the ovarian tissue samples. JFM contributed to the design and coordination of the study and com- posed the final draft of the manuscript. All authors read and approved the final manuscript.

\section{Additional material}

\section{Additional file 1}

Overlap between genes displaying a significant $(p<0.01)$ changes of expression in OVCAR-3 after 5-aza-dC treatment and genes down-regulated in ovarian cancer tissue samples. Fold change in gene expression is shown for 5-aza-dC treated relative to untreated OVCAR 3 cells and for ovarian cancer (EOC) relative to control (NOSE) patient samples. Also shown is the chromosomal location of each gene (Chr).

Click here for file

[http://www.biomedcentral.com/content/supplementary/14764598-6-10-S1.doc]

\section{Acknowledgements}

We are grateful to Kim Totten and Nina Schubert for valuable assistance in the collection of tissue samples. This research was supported by grants from the Georgia Cancer Coalition.

\section{References}

I. Crijns AP, Duiker EW, de Jong S, Willemse PH, van der Zee AG, de Vries EG: Molecular prognostic markers in ovarian cancer: toward patient-tailored therapy. Int J Gynecol Cancer 2006, I6(Suppl I):|152-165.

2. Esteller M: Epigenetics provides a new generation of oncogenes and tumour-suppressor genes. $\mathrm{Br} J$ Cancer 2006, 94:179-183.

3. Herman JG, Baylin SB: Gene silencing in cancer in association with promoter hypermethylation. N Engl J Med 2003, 349:2042-2054.

4. Gardiner-Garden M, Frommer M: CpG islands in vertebrate genomes. I Mol Biol 1987, 196:261-282.

5. Ibanez de Caceres I, Battagli C, Esteller M, Herman JG, Dulaimi E, Edelson MI, Bergman C, Ehya H, Eisenberg BL, Cairns P: Tumor cellspecific BRCAI and RASSFIA hypermethylation in serum, plasma, and peritoneal fluid from ovarian cancer patients. Cancer Res 2004, 64:6476-648I.

6. Makarla PB, Saboorian MH, Ashfaq R, Toyooka KO, Toyooka S, Minna JD, Gazdar AF, Schorge JO: Promoter hypermethylation profile of ovarian epithelial neoplasms. Clin Cancer Res 2005, I I:5365-5369.

7. Teodoridis JM, Hall J, Marsh S, Kannall HD, Smyth C, Curto J, Siddiqui N, Gabra H, McLeod HL, Strathdee G, Brown R: CpG island methylation of DNA damage response genes in advanced ovarian cancer. Cancer Res 2005, 65:896I-8967.

8. Lodygin D, Epanchintsev A, Menssen A, Diebold J, Hermeking $\mathrm{H}$ : Functional epigenomics identifies genes frequently silenced in prostate cancer. Cancer Res 2005, 65:42 18-4227.

9. Mund C, Hackanson B, Stresemann C, Lubbert M, Lyko F: Characterization of DNA demethylation effects induced by 5-Aza2'-deoxycytidine in patients with myelodysplastic syndrome. Cancer Res 2005, 65:7086-7090.

10. Suzuki H, Gabrielson E, Chen W, Anbazhagan R, van Engeland M, Weijenberg MP, Herman JG, Baylin SB: A genomic screen for genes upregulated by demethylation and histone deacetylase inhibition in human colorectal cancer. Nat Genet 2002, $31: 141-149$

II. Takai N, Kawamata N, Walsh CS, Gery S, Desmond JC, Whittaker S, Said JW, Popoviciu LM, Jones PA, Miyakawa I, Koeffler HP: Discovery of epigenetically masked tumor suppressor genes in endometrial cancer. Mol Cancer Res 2005, 3:26I-269.

12. Rivenbark AG, Jones WB, Risher JD, Coleman WB: DNA Methylation-Dependent Epigenetic Regulation of Gene Expression in MCF-7 Breast Cancer Cells. Epigenetics 2006, I:e I-I2. 
13. Wang Z, Zhang J, Zhang Y, Lim SH: SPAN-Xb expression in myeloma cells is dependent on promoter hypomethylation and can be upregulated pharmacologically. Int J Cancer 2006, I I 8: |436-| 444.

14. De Smet C, Lurquin C, Lethe B, Martelange V, Boon T: DNA methylation is the primary silencing mechanism for a set of germ line- and tumor-specific genes with a CpG-rich promoter. Mol Cell Biol 1999, 19:7327-7335.

15. Sunaga N, Miyajima K, Suzuki M, Sato M, White MA, Ramirez RD, Shay JW, Gazdar AF, Minna JD: Different roles for caveolin-I in the development of non-small cell lung cancer versus small cell lung cancer. Cancer Res 2004, 64:4277-4285.

16. Sigalotti L, Coral S, Nardi G, Spessotto A, Cortini E, Cattarossi I, Colizzi F, Altomonte M, Maio M: Promoter methylation controls the expression of MAGE2, 3 and 4 genes in human cutaneous melanoma. J Immunother 2002, 25:16-26.

17. Alaminos M, Davalos V, Ropero S, Setien F, Paz MF, Herranz M, Fraga MF, Mora J, Cheung NK, Gerald WL, Esteller M: EMP3, a myelinrelated gene located in the critical $19 \mathrm{q} / 3.3$ region, is epigenetically silenced and exhibits features of a candidate tumor suppressor in glioma and neuroblastoma. Cancer Res 2005, 65:2565-257।.

18. Cameron EE, Bachman KE, Myohanen S, Herman JG, Baylin SB: Synergy of demethylation and histone deacetylase inhibition in the re-expression of genes silenced in cancer. Nat Genet 1999, 21:103-107.

19. Vanaja DK, Cheville JC, Iturria SJ, Young CY: Transcriptional silencing of zinc finger protein 185 identified by expression profiling is associated with prostate cancer progression. Cancer Res 2003, 63:3877-3882.

20. Chen ST, Lin SY, Yeh KT, Kuo SJ, Chan WL, Chu YP, Chang JG: Mutational, epigenetic and expressional analyses of caveolinI gene in breast cancers. Int J Mol Med 2004, I 4:577-582.

21. Yu Y, Xu F, Peng H, Fang X, Zhao S, Li Y, Cuevas B, Kuo WL, Gray JW, Siciliano M, et al.: NOEY2 (ARHI), an imprinted putative tumor suppressor gene in ovarian and breast carcinomas. Proc Natl Acad Sci USA 1999, 96:2/4-219.

22. Venter JC, Adams MD, Myers EW, Li PW, Mural RJ, Sutton GG, Smith $\mathrm{HO}$, Yandell M, Evans CA, Holt RA, et al.: The sequence of the human genome. Science 200I, 29 I:|304-135|.

23. Jones PA, Laird PW: Cancer epigenetics comes of age. Nat Genet 1999, 21:163-167.

24. Ducasse M, Brown MA: Epigenetic aberrations and cancer. Mol Cancer 2006, 8(5):60.

25. Plass C, Smiraqlia DJ: Genome-wide analysis of DNA methylation changes in human malignancies. Curr Top Microbiol Immunol 2006, 310:179-198.

26. Dannenberg LO, Edenberg HJ: Epigenetics of gene expression in human hepatoma cells: and histone deacetylation. $B M C$ Genomics 2006, 7:181.

27. Yuan BZ, Jefferson AM, Popescu NC, Reynolds SH: Aberrant gene expression in human non small cell lung carcinoma cells exposed to demethylating agent 5-aza-2'-deoxycytidine. Neoplasia 2004, 6:412-419.

28. Yamashita S, Tsujino Y, Moriquchi K, Tatematsu M, Ushijima T: Chemical genomic screening for methylation-silenced genes in gastric cancer cell lines using 5-aza-2'-deoxycytidine treatment and oligonucleotide microarray. Cancer Sci 2006, 97:64-7I.

29. Tusher VG, Tibshirani R, Chu G: Significance analysis of microarrays applied to the ionizing radiation response. Proc Natl Acad Sci USA 2001, 98:5I I6-5I 21 .

30. [http://www.affymetrix.com/analysis/index.affx]. http.//www.molecular-cancer.com/content/6/1/10 